\title{
CRT 玻璃高温自蔓延反应过程中铅的挥发及纳米晶化规律研究
}

\author{
王昱, 谢毅君, 高 雅, 朱建新 \\ (中国科学院生态环境研究中心, 北京 100085)
}

摘 要: 研究了废旧阴极射线管(CRT)玻璃高温自蔓延技术制备纳米 $\mathrm{PbO}$ 的基本过程和规律, 考察了 $\mathrm{Fe}_{2} \mathrm{O}_{3}-\mathrm{Mg}-\mathrm{CRT}$ 自蔓延体系的最高燃烧温度及燃烧波传播速率，探索了不同 $\mathrm{CRT}$ 玻璃配比对铅提取率、物相组成以及微观形貌的 影响, 分析了高温自蔓延反应过程中铅的挥发及纳米晶化规律, 并对 CRT 高温自蔓延反应过程中的热力学机理和 铅的挥发机制进行了初步研究. 实验结果表明, 随着 CRT 玻璃加入量的增加, 高温自蔓延反应的最高燃烧温度、燃 烧波传播速率和产物纳米 $\mathrm{PbO}$ 颗粒的团聚程度均呈减小的趋势. 当 $\mathrm{CRT}$ 玻璃加入量为 $40 \mathrm{wt} \%, \mathrm{CRT}$ 中铅的回收率 约为 $93 \%$, 产物为球状的 $\mathrm{PbO}$ 纳米颗粒, 粒径 40 50 nm. 本研究可以为 CRT 高温自蔓延处理技术的工业化应用提 供一定的理论依据和数据基础.

关 键 词: 废旧阴极射线管玻璃; 高温自蔓延技术; $\mathrm{PbO}$; 纳米颗粒

中图分类号: TQ174 文献标识码: A

\section{Lead Evaporation from CRT Glass and Nanocrystallization Mechanism in the High-temperature Self-propagating Process}

\author{
WANG Yu, XIE Yi-Jun, GAO Ya, ZHU Jian-Xin \\ (Research Center for Eco-Environmental Sciences, Chinese Academy of Sciences, Beijing 100085, China)
}

\begin{abstract}
Lead evaporation and recovery of nanoparticle materials from waste cathode-ray tube (CRT) glass using the self-propagating high-temperature synthesis (SHS) method was investigated. The reaction temperature and combustion velocity of the mixture of $\mathrm{Fe}_{2} \mathrm{O}_{3}-\mathrm{Mg}$-CRT in the SHS process were studied in detail. Then the lead extraction ratio, phase compositions as well as the micromorphology of extracted $\mathrm{PbO}$ nanoparticles were examined. The thermodynamics of lead evaporation and nanocrystallization mechanism were also analyzed. It can be found that the SHS reaction temperature and combustion velocity decrease with increasing CRT glass content, and the addition of CRT glass as a diluent can be used to control the SHS lead extraction behavior to obtain $\mathrm{PbO}$ nanoparticles with good desperation and morphology. With $40 \mathrm{wt} \%$ CRT glass addition, about $93 \%$ of lead can be recovered from CRT glass as nano-sized $\mathrm{PbO}$ with high purity. The collected $\mathrm{PbO}$ nanoparticles are almost spherical in shape with particle sizes ranging from $40 \mathrm{~nm}$ to $50 \mathrm{~nm}$, which are mainly from the volatilization and rapid condensation during the SHS process. The study indicates a novel method to recycle and reuse waste CRT glass.
\end{abstract}

Key words: waste CRT glass; SHS; PbO; nanoparticles

电子废弃物是目前世界上增长最快的固体废弃 物之一. 目前中国个人电脑和电视机的年废弃数量
已经超过了 1000 多万台. 并且, 2009 年开始实施的 家电 “以旧换新” 政策也加速了旧家电的淘汰, 仅

收稿日期: 2011-12-26; 收到修改稿日期：2012-03-21

基金项目：国家自然科学基金 $(20977105,50708110)$ National Natural Science Foundation of China $(20977105,50708110)$

作者简介: 王 昱(1986-), 男, 硕士研究生. E-mail: 10wangyu@gmail.com

通讯作者: 朱建新, 副研究员. E-mail: zhujx@rcees.ac.cn 
在 2009 年 8 月至 2010 年 5 月期间, 回收的废旧 CRT 电脑和电视机的数量就分别达到 59.4 万台和 1206.3 万台 ${ }^{[1-2]}$. 废弃的 CRT 玻璃含有约 $20 \% \sim 30 \%$ 氧化铅, 如不加以妥善回收或处置, 将会对自然环境和人类 健康造成很大的危害 ${ }^{[3]}$.

$\mathrm{CRT}$ 玻璃中铅的分离和回收技术是目前 CRT 玻璃资源化和再生利用的热点和难点. 研究表明, 铅在 CRT 玻璃中扮演着网络中间体的角色, $\mathrm{PbO}_{3}$ 多 面体被 $\mathrm{SiO}_{4}$ 四面体紧紧地包裹而构成连续的三维 网状结构 ${ }^{[4-6]}$. 因此, CRT 的玻璃态稳定结构对铅的 分离造成了困难. Yot 等 ${ }^{[7]}$ 采用碳化硅作为还原剂, 采用 $950^{\circ} \mathrm{C}$ 的高温, 处理 $1 \mathrm{~h}$, 铅的回收率约为 $40 \%$. 虽然目前也有一些学者将 CRT 玻璃高温处理制备 陶瓷和釉质材料, 但由于相关产品中含有大量的铅, 仍具有较大的环境风险, 废旧 CRT 玻璃的处理成为 中国电子废弃物处理处置的关键问题之一.

高温自蔓延反应技术(SHS)具有反应时间短、能 源利用率高和处理过程经济等优点, 在超高温材料 合成、金属间化合物的制造以及粉末冶金工程中有 着广泛的应用. 同时, 高温自蔓延反应温度高、升温 速度快、装置简单和反应一经点燃就不需要任何形 式的外在能源的特点, 在废物处理技术方面具有较 强的优势. 2001 年, Sannia 等 ${ }^{[8]}$ 使用高温自蔓延技术 成功处理含锌废物. 郭志锰等 ${ }^{[9]}$ 也采用高温自蔓延 技术合成 $\mathrm{SrTiO}_{3}$, 处理核废物.

本工作主要研究了废弃 CRT 玻璃高温自蒠延制 备纳米 $\mathrm{PbO}$ 的可行性, 考察了 $\mathrm{Fe}_{2} \mathrm{O}_{3}-\mathrm{Mg}-\mathrm{CRT}$ 自蔓延 体系的热动力学特性, 探索了不同 CRT 玻璃配比对 铅的分离率、物相组成以及纳米 $\mathrm{PbO}$ 微观形貌的影 响，分析了高温自蔓延反应过程中铅的挥发机理.

\section{1 实验}

试验所用 CRT 玻璃取自废弃的 CRT 彩色显示 器中的雉玻璃, 其主要的化学组成如表 1 所示. 首 先将 CRT 玻璃用行星式球磨机破碎并过 $\phi 187.5 \mu \mathrm{m}$ 筛网, 然后将所得 CRT 玻璃粉末放置于烘箱中, 在 $105^{\circ} \mathrm{C}$ 下干燥 $24 \mathrm{~h}$. 实验中所采用的自蔓延反应剂 为分析纯的 $\mathrm{Fe}_{2} \mathrm{O}_{3}$ 和 $\mathrm{Mg}$ (摩尔比 1:3), CRT 玻璃的加 入量为样品总质量的 $10 \mathrm{wt} \% \sim 70 \mathrm{wt} \%$.

取 $5 \mathrm{~g}$ 按一定比例均匀混合的 CRT 玻璃、 $\mathrm{Fe}_{2} \mathrm{O}_{3}$ 和 $\mathrm{Mg}$ 粉末压制坏体, 制坏压力 $10 \mathrm{MPa}$, 时间 $2 \mathrm{~min}$, 坏体尺寸约为 $40 \mathrm{~mm} \times 8 \mathrm{~mm} \times 7 \mathrm{~mm}$. 自曼延反应时, 将坏体坚立放置在反应仓内, 采用铇丝通电加热, 电流 10 15 A, 点火时间 2 3 s. 分别利用红外测温枪 (Raytek 3i) 和电子秒表测定和记录自蔓延反应过
表 1 试验所用 CRT 玻璃化学成分的 XRF 分析

Table 1 Chemical compositions of investigated CRT glass tested by XRF

\begin{tabular}{cc}
\hline Composition & wt $\%$ \\
\hline $\mathrm{SiO}_{2}$ & $52.45 \pm 2.01$ \\
$\mathrm{PbO}$ & $22.89 \pm 0.91$ \\
$\mathrm{~K}_{2} \mathrm{O}$ & $8.66 \pm 0.62$ \\
$\mathrm{Na}_{2} \mathrm{O}$ & $5.67 \pm 0.25$ \\
$\mathrm{Al}_{2} \mathrm{O}_{3}$ & $3.68 \pm 0.04$ \\
$\mathrm{CaO}$ & $2.74 \pm 0.31$ \\
$\mathrm{MgO}$ & $2.43 \pm 0.66$ \\
\hline
\end{tabular}

程中最高燃烧温度及燃烧波传播速率.

高温自蔓延反应完成后得到两种产物：纳米 $\mathrm{PbO}$ 颗粒(挥发冷凝物)和燃烧后固体剩余物. 产物 的物相鉴定采用 X 射线衍射分析 (XRD, $\chi$ 'Pert PRO MPD, PANalytical, Netherlands); 样品中 $\mathrm{Pb}$ 元素的含 量采用美国环保署 SW846-3050 消解程序完全消解, 并用 ICP-OES(OPTIMA 2000, PerkinElmer, U. S.)测 定; 收集到的纳米 $\mathrm{PbO}$ 颗粒首先放置于无水乙醇中 使用超声波将粉末分散, 然后使用透射电镜(TEM, H-7500, Hitachi, Japan)分析. 剩余固体样品的环境 浸出毒性按照美国环保署固体废物 SW804-1311 毒 性浸出鉴别程序进行实验评价.

\section{2 结果及分析}

\section{1 CRT 高温自蔓延燃烧波传播规律}

$\mathrm{Fe}_{2} \mathrm{O}_{3} 、 \mathrm{Mg}$ 和 $\mathrm{CRT}$ (CRT 玻璃的简称，以下同) 的高温自蔓延实验结果表明, 当 CRT 的加入量为 $10 \mathrm{wt} \% \sim 60 \mathrm{wt} \%$ 时，体系可以维持自蔓延反应. 当 CRT 加入量为 $10 \mathrm{wt} \% \sim 30 \mathrm{wt} \%$, 体系的高温自蔓延 反应极为剧烈, 燃烧过程会发生强烈的熔质飞溅; 同时, 样品有明显的熔融和塌陷现象. 当 CRT 加入 量为 $40 \mathrm{wt} \% \sim 60 \mathrm{wt} \%$ 时, 燃烧速度均匀, 反应过程平 稳, 无明显飞溅发生, 样品形状完整.

图 1(a)是 $\mathrm{Fe}_{2} \mathrm{O}_{3} 、 \mathrm{Mg}$ 和 CRT 高温自蔓延反应的 最高燃烧温度与 CRT 加入量的关系图, 从图中可以 看出随着 CRT 加入量的增加, 系统的最高燃烧温度 呈降低趋势. 当 CRT 加入量为 $10 \mathrm{wt} \%$ 时, 系统的最 高燃烧温度为 $2765 \mathrm{~K}$; 当 CRT 加入量为 $60 \mathrm{wt} \%$ 时, 最高燃烧温度下降到 $1527 \mathrm{~K}$.

图 1(b) 是 $\mathrm{Fe}_{2} \mathrm{O}_{3} 、 \mathrm{Mg}$ 和 CRT 高温自蔓延反应燃 烧波传播速率与 $\mathrm{CRT}$ 加入量的关系图, 从图中可以 看出, 随着 CRT 加入量的增大, 燃烧波的传播速率 呈降低趋势. 当 CRT 加入量为 $10 \mathrm{wt} \%$ 时, 燃烧波的 平均传播速率高达 $23.6 \mathrm{~mm} / \mathrm{s}$; CRT 加入量为 

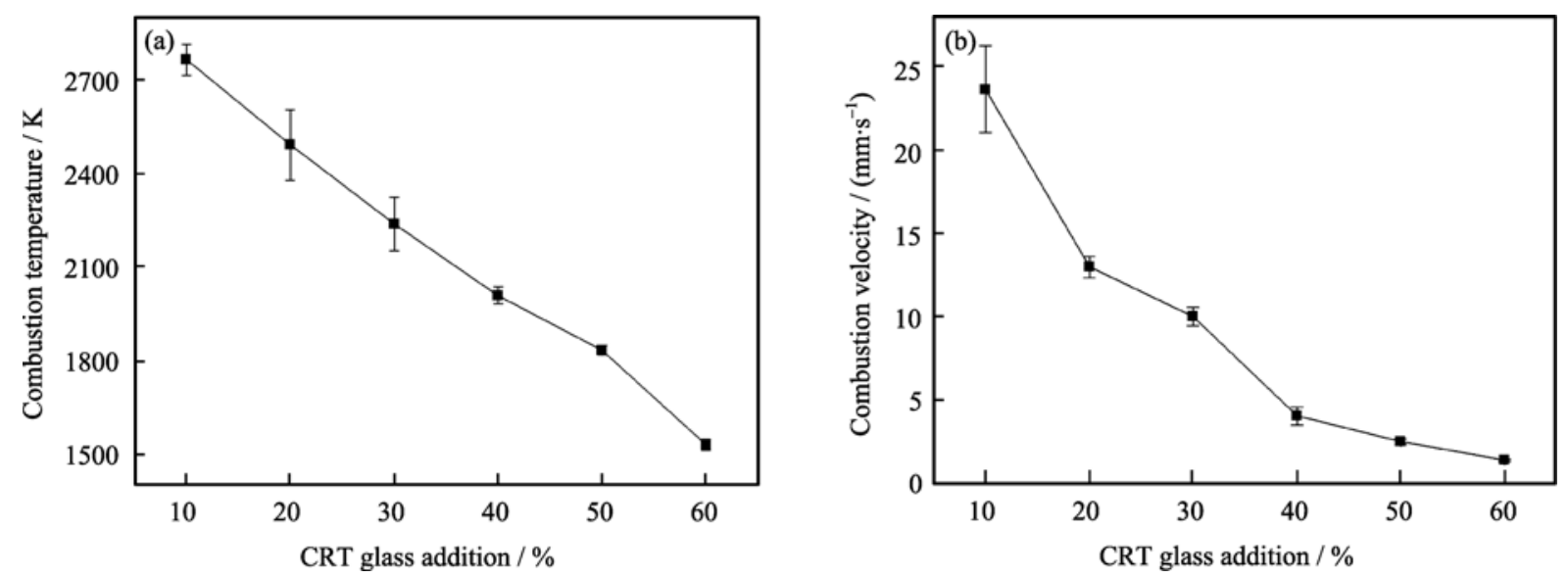

图 1 CRT 加入量对(a)最高燃烧温度 $\left(T_{\mathrm{c}}\right)$ 和(b)燃烧波传播速率的影响

Fig. 1 Effect of CRT addition in the original mixture on (a) maximum combustion temperature and (b) the combustion velocity

$20 \mathrm{wt} \%$ 30wt \% 时，燃烧波传播速率迅速降低至 $10 \sim 13 \mathrm{~mm} / \mathrm{s}$; CRT 加入量为 $40 \mathrm{wt} \% \sim 60 \mathrm{wt} \%$ 时, 燃烧 波的传播速率维持在 $5 \mathrm{~mm} / \mathrm{s}$ 以下.

当 CRT 加入量为 $70 \mathrm{wt} \%$ 时, 使用铇丝加热 $5 \mathrm{~s}$ 时也不能点燃压坏样品. 从实验结果可以看出, 使 用单位质量的 $\mathrm{Fe}_{2} \mathrm{O}_{3}$ 和 $\mathrm{Mg}$ 自蔓延剂最多约可以处 理 1.5 倍质量的 CRT 废物.

\section{2 CRT 高温自蔓延反应铅的分离规律}

表 2 是 $\mathrm{Fe}_{2} \mathrm{O}_{3} 、 \mathrm{Mg}$ 和 CRT 高温自蔓延反应氧 化铅挥发量与 $\mathrm{CRT}$ 加入量的关系表, 从表中可以看 出当 CRT 加入量为 $10 \mathrm{wt} \% \sim 40 \mathrm{wt} \%$ 时，高温自蔓延 反应过程中氧化铅的回收率在 $93 \%$ 以上. 当 CRT 加 入量为 $50 \mathrm{wt} \%$ 时，氧化铅的回收率约为 $78 \%$. 但当 $\mathrm{CRT}$ 加入 $60 \mathrm{wt} \%$ 时，仅有约 $12 \%$ 的氧化铅挥发和分 离出来.

图 2 是不同 CRT 加入量高温自蔓延反应挥发凝 结物的 XRD 图谱. 从图 2 可以看出, 当 CRT 加入量 为 $40 \mathrm{wt} \%$ 及以上时, 挥发冷凝物的物相主要为 $\mathrm{PbO}$. 而当 CRT 加入量为 $10 \mathrm{wt} \%$ 30wt\%时，挥发冷凝物的 物相组成比较复杂, 除了主要物相 $\mathrm{PbO}$ 外, 还有 $\mathrm{MgO} 、 \mathrm{Fe}_{2} \mathrm{O}_{3}$ 和 $\mathrm{SiO}_{2}$ 等物相存在. 分析可知, 当 CRT 加入量为 $10 \mathrm{wt} \% \sim 30 \mathrm{wt} \%$ 时，自蔓延反应的最高燃烧

表 2 不同 CRT 加入量时 PbO 的分离效率

Table 2 Extraction ratio of $\mathrm{PbO}$ on CRT addition

\begin{tabular}{ccccc}
\hline No & $\begin{array}{c}\mathrm{CRT} \\
/ \mathrm{wt} \%\end{array}$ & $\begin{array}{c}\mathrm{Mg} \\
/ \mathrm{wt} \%\end{array}$ & $\begin{array}{c}\mathrm{Fe}_{2} \mathrm{O}_{3} \\
/ \mathrm{wt} \%\end{array}$ & $\begin{array}{c}\text { PbO evapo- } \\
\text { rated/wt\% }\end{array}$ \\
\hline CRT10 & 10.0 & 28.2 & 61.8 & $94 \pm 0.7$ \\
CRT20 & 20.0 & 25.1 & 54.9 & $95 \pm 0.7$ \\
CRT30 & 30.0 & 21.9 & 48.1 & $95 \pm 0.3$ \\
CRT40 & 40.0 & 18.8 & 41.2 & $93 \pm 0.5$ \\
CRT50 & 50.0 & 15.7 & 34.3 & $78 \pm 1.5$ \\
CRT60 & 60.0 & 12.5 & 27.5 & $12 \pm 3.6$ \\
\hline
\end{tabular}

温度约为 2237 2765 K 之间, 远远超过 $\mathrm{PbO}$ 的沸点但 低于 $\mathrm{SiO}_{2}$ 的沸点 $(3223 \mathrm{~K})$ 及 $\mathrm{MgO}$ 的沸点 $(3873 \mathrm{~K})^{[10] \text {, }}$ 因此当 CRT 加入量较低时, $\mathrm{SiO}_{2} 、 \mathrm{Fe}_{2} \mathrm{O}_{3}$ 和 $\mathrm{MgO}$ 主要 来自反应过程发生的火焰飞溅，而不是由挥发产生 的. 当 CRT 加入量加大时, 燃烧比较稳定, 无明显飞 贼现象发生, 所以产物 $\mathrm{PbO}$ 纯度较高.

图 3 是 $\mathrm{Fe}_{2} \mathrm{O}_{3} 、 \mathrm{Mg}$ 和 CRT 体系高温自蔓延反 应完成后固体剩余物的 XRD 图谱, 从图中可以看 出, 残余相的主要物相为 $\mathrm{Mg}_{2} \mathrm{SiO}_{4} 、 \mathrm{MgO} 、 \mathrm{Fe}$, 表明 高温自蔓延反应的主要放热反应为:

$$
3 \mathrm{Mg}+\mathrm{Fe}_{2} \mathrm{O}_{3}+3 \mathrm{SiO}_{2}=\mathrm{Mg}_{2} \mathrm{SiO}_{4}+\mathrm{MgO}+2 \mathrm{Fe}
$$

表 3 是高温自曼延处理前后 CRT 样品的环境毒 性浸出实验结果, 可以看出高温自蔓延可以实现废 弃 CRT 的高效无害化, 处理后固体剩余物的毒性浸 出浓度从原始 CRT 的 $72.5 \mathrm{mg} / \mathrm{L}$ 降低到 0.02 0.18 mg/L. 原始的 CRT 浸出浓度高出危险废物环境浸出毒性 标准 10 倍以上, 处理时需要对 $\mathrm{Pb}$ 固化稳定化后进 入专门的危险废物填埋场. 而经高温自蔓延处理后,

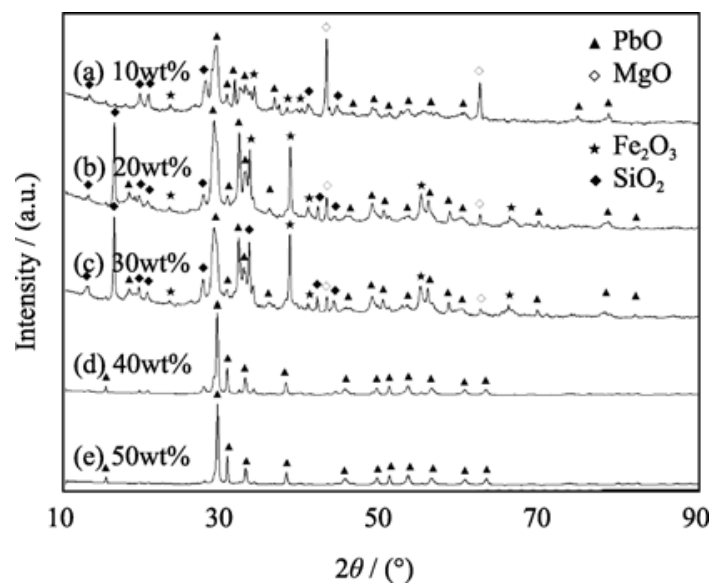

图 2 不同 $\mathrm{CRT}$ 加入量时, 挥发凝结物的 XRD 图谱 Fig. 2 XRD patterns of prepared powders with various CRT additions in the original mixture 


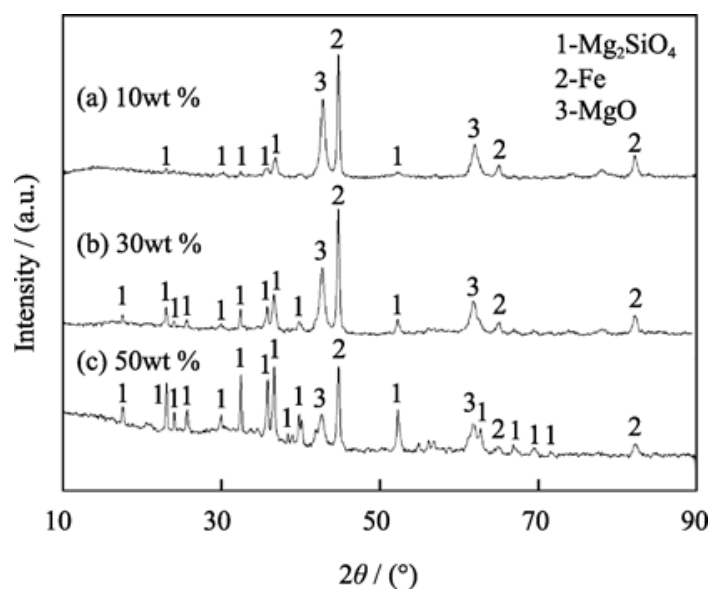

图 3 不同 $\mathrm{CRT}$ 加入量时, 固体剩余物的 XRD 图谱

Fig. 3 XRD patterns of residues with various CRT additions in the original mixture

样品完全可以作为普通废物处理或进行资源化利用.

\section{3 纳米氧化铅的微观形貌}

图 4 是 CRT 加入量分别为 $10 \mathrm{wt} \%$ 和 $40 \mathrm{wt} \%$ 时, 经高温自蔓延处理得到的纳米 $\mathrm{PbO}$ 颗粒的 TEM 照 片. 从图 4 可以看出, 高温自蔓延产生的快速加热 和急速冷却条件, 有利于形成形状规则的球形纳米

表 3 高温自蔓延处理前后 CRT 样品的毒性浸出浓度

Table 3 TCLP results of lead with various CRT additions after SHS treatment

\begin{tabular}{cc}
\hline Sample & $\begin{array}{c}\mathrm{Pb} \text { concentration in TCLP } \\
/\left(\mathrm{mg} \cdot \mathrm{L}^{-1}\right)\end{array}$ \\
\hline CRT & 72.5 \\
CRT10 & 0.10 \\
CRT20 & 0.09 \\
CRT30 & 0.18 \\
CRT40 & 0.02 \\
CRT50 & 0.07 \\
CRT60 & 0.10 \\
Regulory limitation & 5.00 \\
\hline
\end{tabular}

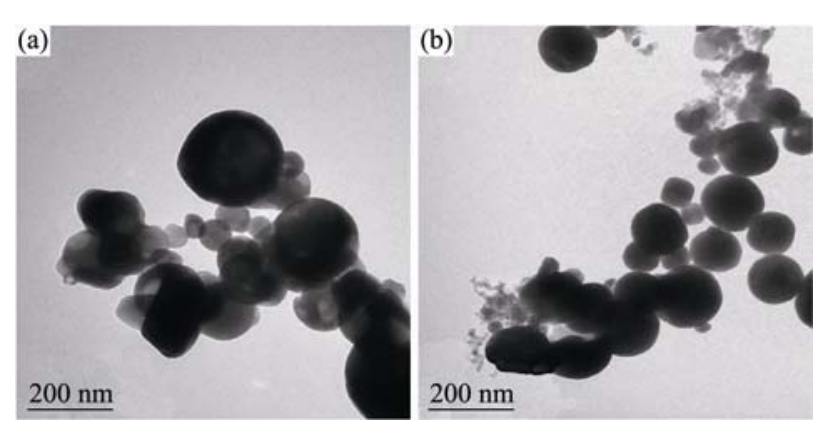

图 4 不同 CRT 加入量以及最高燃烧温度条件下合成的纳 米 $\mathrm{PbO}$ 颗粒的 TEM 照片

Fig. 4 Typical TEM images of the as-prepared $\mathrm{PbO}$ nanoparticles with different CRT additions at different combustion temperatures (a) $\mathrm{CRT}=10 \mathrm{wt} \%, T_{\mathrm{c}}=2765 \mathrm{~K}$; (b) $\mathrm{CRT}=40 \mathrm{wt} \%, T_{\mathrm{c}}=2008 \mathrm{~K}$
$\mathrm{PbO}$ 颗粒，尺寸介于 $40 \sim 50 \mathrm{~nm}$ 之间. 该类纳米 $\mathrm{PbO}$ 可以作为重要的工业原料用于制造燃速催化剂、化 学电源的活性物质、高折射率的光学玻璃等方面, 具有较高的潜在商业价值.

从图 4 中还可以看出, 增加 CRT 加入量, 纳米 $\mathrm{PbO}$ 的团聚程度呈降低趋势, 当 $\mathrm{CRT}$ 加入量为 $40 \mathrm{wt} \%$, 得到分散程度较好的纳米 $\mathrm{PbO}$ 颗粒. 这主要是由于当 CRT 加入量较小时, SHS 燃烧温度较高, $\mathrm{PbO}$ 的挥发速 率相对较快, 单位时间内颗粒之间的碰撞和结合频率 越高, 这导致了较大团聚体的形成 ${ }^{[11-13]}$.

结合表 2 和图 2 对照图 4 的表观形貌分析结果 可知: 当 CRT 加入量为 $40 \mathrm{wt} \%$, 高温自蔓延过程结 果较优, 不但可以得到纯度较高和形状规则的纳米 $\mathrm{PbO}$ 颗粒，而且可以实现相对较高的废物处理量.

高温自蔓延过程中 CRT 玻璃中氧化铅的挥发 和冷凝过程主要有两种形式: 一种形式是在高温自 蔓延反应过程中快速挥发和冷凝形成纳米 $\mathrm{PbO}$ 颗粒; 另一种形式是高温自蔓延反应首先使 CRT 成为过 热液体, 而反应后过热液体中的 $\mathrm{PbO}$ 逐渐挥发和冷 凝形成 $\mathrm{PbO}$ 纳米颗粒. 根据过热液体的闪蒸理论, 过热液体的闪蒸率可用下式表示:

$$
\eta_{\max }=C_{\mathrm{p}} \frac{T_{\mathrm{c}}-T_{\mathrm{b}}}{H_{\mathrm{v}}}
$$

其中 $\eta_{\text {max }} 、 C_{\mathrm{p}} 、 T_{\mathrm{b}}$ 和 $H_{\mathrm{v}}$ 分别为过热液体的闪蒸率、 热容、闪蒸物质的沸点和蒸发潜热. $T_{\mathrm{c}}$ 是过热液体温 度, 在本研究中为自蔓延反应实际燃烧温度.

图 5 是当高温自蔓延初始混合物中 CRT 玻璃的 含量为 $10 \mathrm{wt} \% \sim 40 \mathrm{wt} \%$ 时铅的理论闪蒸率，由图可知 铅的理论闪蒸率只占实际提取率的 $8.96 \%$ 34.23\%. 这表明高温自蔓延反应的铅提取过程即纳米 $\mathrm{PbO}$ 主

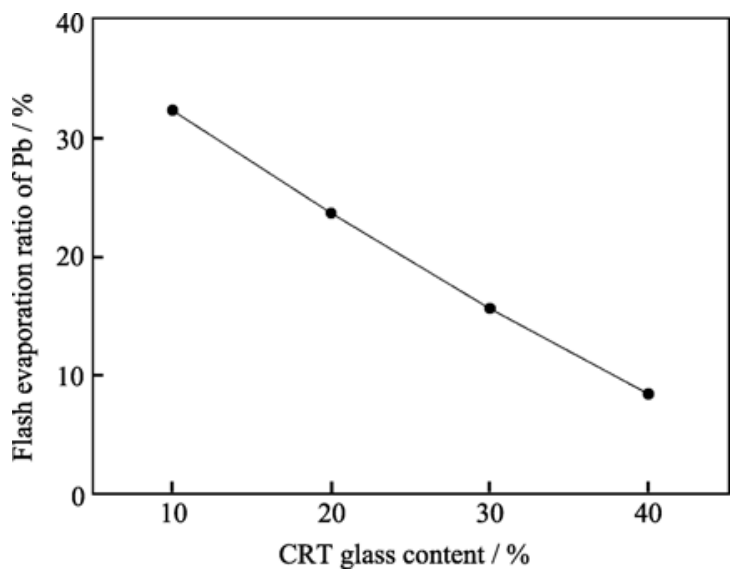

图 5 高温自蔓延初始混合物中 CRT 的含量为 $10 \mathrm{wt} \%$ $40 \mathrm{wt} \%$ 时铅的理论闪蒸率

Fig. 5 Flash evaporation ratio of $\mathrm{Pb}$ with various $\mathrm{CRT}$ additions ranged from $10 \mathrm{wt} \%$ to $40 \mathrm{wt} \%$ 
要是在高温自蔓延反应过程中挥发并快速冷凝完成 的，具有工艺简单和生产效率高的优点.

\section{4 反应机理研究}

反应过程中的活化能是高温自蔓延反应特性分 析的重要依据. 当假设该高温自蔓延体系的燃烧波 为稳态燃烧, 则可根据 Khachin 导出的在稳态燃烧 条件下的燃烧速率 $v$ 的表达式计算 ${ }^{[14]}$ :

$$
v^{2}=f(n) \frac{a C_{\mathrm{p}}}{Q} \frac{R T_{\mathrm{c}}^{2}}{E} K_{0} \exp \left(-E / \mathrm{R} T_{\mathrm{c}}\right)
$$

其中 $a, T_{\mathrm{c}}, C_{\mathrm{p}}, Q$ 和 $E$ 分别为导热系数、最高燃烧温 度、定压热容、反应热和反应活化能. $f(n)$ 为反应级 数为 $n$ 的函数, 假设本反应为一级反应, 即 $n=1$, $f(n)=1$.

通过实验测出不同最高燃烧温度下的燃烧速 率, 作出 $\ln \left(v / T_{\mathrm{c}}\right) \sim 1 / T_{\mathrm{c}}$ 图, 如图 6 所示. 由图 6 可以 看出, 该高温自蔓延体系的反应活化能呈现两段 式变化趋势，这表明该高温自蔓延体系在不同燃 烧温度条件下的燃烧机理不同. 实验现象也表明 随着初始混合物中 CRT 加入量的不同, 该高温自 蔓延过程呈现不同的燃烧模式. 根据斜率计算图 中两个不同斜率对应的反应活化能分别为 165.9 和 $81.7 \mathrm{~kJ} / \mathrm{mol}$, 两个过程的速控步骤分别为化学反应 控制和扩散控制.

$\mathrm{Fe}_{2} \mathrm{O}_{3} 、 \mathrm{Mg}$ 和 $\mathrm{CRT}$ 高温自莫延反应的现象与 Porcu 等 ${ }^{[15]}$ 利用高温自蔓延处理石棉的现象和规律 基本一致. 当 CRT 加入量为 $40 \mathrm{wt} \% \sim 60 \mathrm{wt} \%$ 时, CRT 体系反应活化能与石棉体系反应活化能 $58.9 \mathrm{~kJ} / \mathrm{mol}$ 接近，表明两个体系反应机理基本一致，废物处理 过程中的热量均来源于自蔓延剂 $\mathrm{Fe}_{2} \mathrm{O}_{3}$ 与 $\mathrm{Mg}$ 的反 应放热，石棉及 CRT 在自蔓延系统中起的作用主要 是稀释剂.

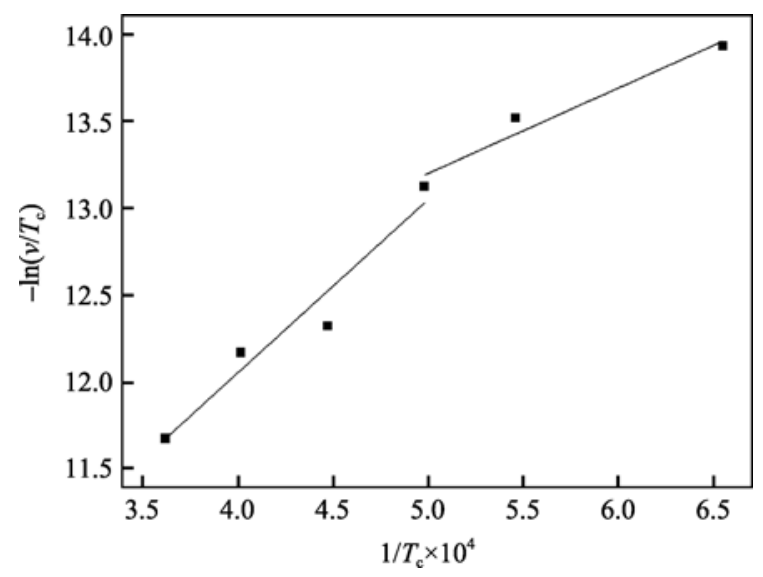

图 6 CRT 高温自蔓延反应的 $\ln \left(v / T_{\mathrm{c}}\right)$ 对 $1 / T_{\mathrm{c}}$ 拟合图

Fig. 6 Plot of $\ln \left(v / T_{\mathrm{c}}\right)$ vs $1 / T_{\mathrm{c}}$ for the SHS treatment of CRT

\section{3 结论}

1) 采用高温自蔓延技术可以实现 CRT 玻璃铅 的高效分离, 生成并回收纳米 $\mathrm{PbO}$. 燃烧剩余的固体 残余物可以作为普通废物处理或资源化回收;

2) $\mathrm{Fe}_{2} \mathrm{O}_{3} 、 \mathrm{Mg}$ 和 CRT 高温自曼延体系中，随着 CRT 加入量的增加, 系统的最高燃烧温度及燃烧波 传播速率均呈降低趋势，废弃 CRT 的作用类似于稀 释剂，不参与燃烧和放热反应;

3) 当 CRT 加入量为 $40 \mathrm{wt} \%$ 时, 铅的回收率约为 $93 \%$, 纳米 $\mathrm{PbO}$ 的尺寸为 $40 \sim 50 \mathrm{~nm}$; 纳米 $\mathrm{PbO}$ 主要 是在高温自暻延反应过程中挥发并快速冷凝完成的.

\section{参考文献:}

[1] Chi X W, Streicher-Porte M, Wang M Y L, et al. Informal electronic waste recycling: a sector review with special focus on China. Waste Manage., 2011, 31(4): 731-742.

[2] Ministry of Environmental Protection of China. Guideline on the Preparation of Development Plan for the WEEE Industry, 2010. http://www. zhb. gov. cn/gkml/hbb/bgg/201011/t20101116_197563. htm.

[3] Lee C H, Hsi C S. Recycling of scrap cathode ray tubes. Environ. Sci. Technol., 2002, 36(1): 69-75.

[4] Witkowska A, Rybicki J, Trzebiatowski K, et al. Influence of hydrogen reduction on the structure of $\mathrm{PbSiO}_{3}$ glass: an EXAFS study. J. Non-Cryst. Solids, 2000, 276(1/2/3): 19-26.

[5] Witkowska A, Rybicki J, Di Cicco A. Structure of partially reduced $x \mathrm{PbO}(1-x) \mathrm{SiO}_{2}$ glasses: combined EXAFS and MD study. J. Non-Cryst. Solids, 2005, 351(5): 380-393.

[6] Mear F O, Yot P G, Kolobov A V, et al. Local structure around lead, barium and strontium in waste cathode-ray tube glasses. $J$. Non-Cryst. Solids, 2007, 353(52/53/54): 4640-4646.

[7] Yot P G, Mear F O. Lead extraction from waste funnel cathoderay tubes glasses by reaction with silicon carbide and titanium nitride. J. Hazard. Mater., 2009, 172(1): 117-123.

[8] Sannia M, Orru R, Concas A, et al. Self-propagating reactions for environmental protection: Remarks on the treatment and recycling of zinc hydrometallurgical wastes. Ind. Eng. Chem. Res., 2001, 40(3): 801-807.

[9] ZHANG Rui-Zhu, GUO Zhi-Meng, GAO Feng. Solidification of HLW into mineral-like materials by SHS method. Chinese Journal of Rare Metals, 2005, 29(1): 25-29.

[10] Speight J G. Lange's Handbook of Chemistry, 16th Edition. New York: McGraw-Hill, 2005.

[11] Turker M. Effect of production parameters on the structure and morphology of $\mathrm{Ag}$ nanopowders produced by inert gas condensation. Mater. Sci. Eng. A-Struct. Mater. Prop. Microstruct. Process., 2004, 367(1/2): 74-81.

[12] Simchi A, Ahmadi R, Reihani S M S, et al. Kinetics and mechanisms of nanoparticle formation and growth in vapor phase condensation process. Mater. Des. , 2007, 28(3): 850-856.

[13] Ehrman S H, Aquino-Class M I, Zachariah M R. Effect of temperature and vapor-phase encapsulation on particle growth and morphology. J. Mater. Res., 1999, 14(4): 1664-1671.

[14] Holt J B, Munir Z A. Combustion synthesis of titanium carbidetheory and experiment. J. Mater. Sci., 1986, 21(1): 251-259.

[15] Porcu M, Orru R, Cincotti A, et al. Self-propagating reactions for environmental protection: treatment of wastes containing asbestos. Ind. Eng. Chem. Res., 2005, 44(1): 85-91. 